\title{
Computational Approach to Gravitational Waves Forms in Stellar Systems as Complex Structures through Keplerian Parameters
}

\author{
Bochicchio Ivana \\ Dipartimento di Matematica e Informatica \\ Universitá degli studi di Salerno \\ Fisciano (SA), Italy \\ and INFN Sez. di Napoli, \\ Napoli, Italy \\ Email: ibochicchio@unisa.it
}

\author{
De Laurentis Mariafelicia \\ Dipartimento di Scienze Fisiche \\ Universitá di Napoli “ Federico II”, \\ and INFN Sez. di Napoli, \\ Napoli, Italy \\ Email:felicia@na.infn.it
}

\author{
Laserra Ettore \\ Dipartimento di Matematica e Informatica \\ Universitá degli studi di Salerno \\ Fisciano (SA), Italy \\ and INFN Sez. di Napoli, \\ Napoli, Italy \\ Email: elaserra@unisa.it
}

\begin{abstract}
In this paper we investigate the gravitational waves emission by stellar dynamical structures as complex systems in the quadrupole approximation considering bounded and unbounded orbits. Precisely, after deriving analytical expressions for the gravitational wave luminosity, the total energy output and gravitational radiation amplitude, we present a computational approach to evaluate the gravitational wave-forms from elliptical, circular, parabolic and hyperbolic orbits as a function of Keplerian parameters.
\end{abstract}

Index Terms-gravitational waves; theory of orbits; numerical gravity;

\section{INTRODUCTION}

In recent years, detailed information has been achieved for kinematics and dynamics of stars moving in the gravitational field of such a central object. The statistical properties of spatial and kinematical distributions are of particular interest. Using them, it is possible to give a quite accurate estimate of the mass and the size of the central object. More precisely, in [1], it is described a campaign of observations where velocity measurements are extremely accurate. Then from this bulk of data, considering a field of resolved stars whose proper motions are accurately known, one can classify orbital motions and deduce, in principle, the rate of production of GWs according to the different types of orbits. This motivates this paper in which, by a classification of orbits in accordance with the conditions of motion, we want to calculate the GW luminosity for the different types of stellar encounters. Following the method outlined in [2], [3], [4], we investigate the GW emission by stellar dynamical structures as complex systems in the quadrupole approximation considering bounded (circular or elliptical) and unbounded (parabolic or hyperbolic) orbits.

The main parameter is the approaching energy of the stars in the system (see also [5] and references therein). However, in a recent work [6], it is shown that the parametric form of the main evolution equation of Lemaitre-Tolman-Bondi (LTB) cosmological model can be obtained considering the limiting rectilinear solutions of Keplers problem. More precisely, the analogy between the relativistic evolution of LTB shells and the classical discussion of a falling body in a Newtonian center of attraction, allows us to conclude that the relativistic orbits are clearly related to suitable astronomic coordinates. Here, these coordinates can be adopted in order to have a detailed investigation of the gravitational waves emission by stellar encounters.

Precisely, briefly recalling the main features of stellar encounters and orbit classification, we present the computational aspect to obtain the gravitational wave-forms for elliptical, parabolic and hyperbolic encounters.

\section{ORbit In STEllar ENCOUNTERS}

Let us take into account the Newtonian theory of orbits since stellar systems, also if at high densities and constituted by compact objects, can be usually assumed in Newtonian regime. We give here a self-contained summary of the well-known orbital types in order to achieve below a clear classification of the possible GW emissions. We refer to the text books [7], [8], [9] for a detailed discussion.

A mass $m_{1}$ is moving in the gravitational potential $\Phi$ generated by a second mass $m_{2}$. The vector radius and the polar angle depend on time as a consequence of the star motion, i.e. $\mathbf{r}=\mathbf{r}(\mathrm{t})$ and $\phi=\phi(t)$. As it is known, the shape of the orbit depends by the total energy $H$ of the system. Precisely

when $H<0$ the orbit is an ellipse and the equation of the trajectory is

$$
r=\frac{l}{1+\varepsilon \cos \phi},
$$

where $l$ is the so-called semi-latus rectum or the parameter of the ellipse and $\varepsilon$ is the eccentricity of the ellipse (for more details see [4]).

In a generic elliptic Keplerian motion, called $\alpha$ the major semiaxis and $E$ the eccentric anomaly, the orbit can be written also as (see [6], [9])

$$
r=\alpha(1-\varepsilon \cos E)
$$


hence, there is the following relation between the eccentric anomaly and the angle $\phi$ :

$$
\cos \phi=\frac{\cos E-\varepsilon}{1-\varepsilon \cos E} .
$$

While $H \geq 0$ is the condition to obtain unbounded orbits. The trajectory is

$$
r=\frac{l}{1+\varepsilon \cos \phi},
$$

where $\varepsilon \geq 1$. The equal sign corresponds to $H=0$. Therefore, in order to ensure positivity of $r$, the polar angle $\phi$ has to be restricted to the range given by

$$
1+\varepsilon \cos \phi>0 \text {. }
$$

This means $\cos \phi>1$, i.e. $\phi \in(-\pi, \pi)$ and the trajectory is not closed any more. For $\phi \rightarrow \pm \pi$, we have $r \rightarrow \infty$. The curve (4), with $\varepsilon=1$, is a parabola. For $\varepsilon>1$, the allowed interval of polar angles is smaller than $\phi \in(-\pi, \pi)$, and the trajectory is a hyperbola. Such trajectories correspond to nonreturning objects (for more details see [4]).

Lets consider a generic Hyperbolic Keplerian Orbit, with eccentricity $\varepsilon$, real semi-axis $\alpha$ and $F$ as variable, analogous to the elliptic eccentric anomaly $E$. The orbit is defined by

$$
r=\alpha(\varepsilon \cosh F-1) ;
$$

hence, there is the following relation between $F$ and the angle $\phi:$

$$
\cos \phi=\frac{l-\alpha(\varepsilon \cosh F-1)}{\varepsilon \alpha(\varepsilon \cosh F-1)} .
$$

Lets consider the Keplerian Parabolic Orbit. It is defined by the relation

$$
r=\frac{P^{2}}{2}
$$

where $\mathrm{P}$ is a parameter. In this case

$$
\cos \phi=\frac{2 l-P^{2}}{P^{2}} .
$$

In the next section we present the computational approach to obtain gravitational wave-forms in the different cases as function of the eccentric anomaly, the angle $F$ and the parameter $P$.

Remark 1. Let the eccentricity tend to unity in equations (2)(7); in the limit the corresponding orbits are called rectilinear ellipse, hyperbola and parabola respectively.

The evolution of the r-shell in LTB models is analogous to a Keplerian motion on a rectilinear ellipse, hyperbola or parabola (for more details see [6]).

\section{Computational Approach to the Gravitational WAVE FORMS}

At this point, considering the orbit equations, we want to classify the gravitational radiation for the different stellar encounters. We send the Reader to the References [10], [11], [12], [13] for a detailed exposition.

The Einstein field equations give a description of how the curvature of space-time is related to the energy-momentum distribution. In the weak field approximation, moving massive objects produce gravitational waves which propagate in the vacuum with the speed of light. One can search for wave solutions of field equation from a system of masses undergoing arbitrary motions, and then obtain the power radiated [4]. The result, assuming the source dimensions very small with respect to the wavelengths (quadrupole approximation [8]), is that the power $\frac{d H}{d \Omega}$ radiated in a solid angle $\Omega$ with tensor of polarization $e_{i j}$ is

$$
\frac{d H}{d \Omega}=\frac{G}{8 \pi c^{5}}\left(\frac{d^{3} Q_{i j}}{d t^{3}} e_{i j}\right)
$$

where $Q_{i j}$ is the quadrupole mass tensor

$$
Q_{i j}=\sum_{a} m_{a}\left(3 x_{a}^{i} x_{a}^{j}-\delta_{i j} r_{a}^{2}\right)
$$

$G$ being the Newton constant, $r_{a}$ the modulus of the vector radius of the a-th particle and the sum running over all masses $m_{a}$ in the system. With this formalism, it is possible to estimate the amount of energy emitted in the form of GWs from a system of massive objects interacting among them [2], [3]. In this case, the components of the quadrupole mass tensor in the equatorial plane $\left(\theta=\frac{\pi}{2}\right)$ are:

$$
\begin{aligned}
& Q_{x x}=\mu r^{2}\left(3 \cos ^{2} \phi-1\right), \\
& Q_{y y}=\mu r^{2}\left(3 \sin ^{2} \phi-1\right), \\
& Q_{z z}=-\mu r^{2}, \\
& Q_{x z}=Q_{z x}=0, \\
& Q_{y z}=Q_{z y}=0, \\
& Q_{x y}=Q_{y x}=3 \mu r^{2} \cos \phi \sin \phi,
\end{aligned}
$$

where $\mu=\frac{m_{1} m_{2}}{m_{1}+m_{2}}$ is the reduced mass of the system and the masses $m_{1}$ and $m_{2}$ have polar coordinates $\left\{r_{i} \cos \theta \cos \phi, r_{i} \cos \theta \sin \phi, r_{i} \sin \theta\right\}$ with $i=1,2$. The origin of the motions is taken at the center of mass.

Direct signatures of gravitational radiation are its amplitude and its wave-form. In other words, the identification of a GW signal is strictly related to the accurate selection of the shape of wave-forms by interferometers or any possible detection tool. Such an achievement could give information on the nature of the GW source, on the propagating medium, and , in principle, on the gravitational theory producing such a radiation [14]. It is well known that the amplitude $h$ of GWs can be evaluated by

$$
h_{j k}(t, R)=\frac{2 G}{R c^{4}} \ddot{Q}_{j k},
$$

$R$ being the distance between the source and the observer and $\{j, k\}=1,2$.

Moreover, in accordance to

$$
h \simeq\left(h_{11}^{2}+h_{22}^{2}+2 h_{12}^{2}\right)^{1 / 2}
$$


we can compute the expected strain amplitude for different orbits.

Note that we are going to study the evolution of compact binary systems that are formed through the capture of a moving mass $m_{1}$ by a gravitational field, whose source is a Black Hole(BK) of mass $m_{2}$ and being $m_{1} \ll m_{2}$. So we will have that the reduced mass of the newborn binary system $\mu \cong m_{1}$ and the mass ratio $\frac{m_{1}}{m_{2}}$ is $\ll 1$. This constraint is satisfied by several real systems.

Let us now derive the GW amplitude in relation to the orbital shape of the binary systems.

\section{A. Quadrupole and GW Amplitude from Elliptical Orbits}

In this section the components of the quadrupole mass tensor in the equatorial plane for the elliptical orbits are expressed. Precisely, by Eq.s (2), (3) and (9), we obtain:

$$
\begin{aligned}
Q_{x x} & =-\alpha^{2} m_{1}\left[-3 \varepsilon^{2}+4 \varepsilon \cos E+\left(\varepsilon^{2}-3\right) \cos ^{2} E+1\right], \\
Q_{y y} & =\alpha^{2} m_{1}\left[-3 \varepsilon^{2}+2 \varepsilon \cos E+\left(2 \varepsilon^{2}-3\right) \cos ^{2} E+2\right], \\
Q_{z z} & =-\alpha^{2} m_{1}(\varepsilon \cos E-1)^{2}, \\
Q_{x y} & =Q_{y x}= \\
& =3 \alpha^{2} m_{1}(\varepsilon-\cos E)(\varepsilon \cos E-1) \sqrt{-\frac{\left(\varepsilon^{2}-1\right) \sin ^{2} E}{(\varepsilon \cos E-1)^{2}}}
\end{aligned}
$$

Moreover, considering a binary system and the single components of Eq. (10), it is straightforward to show that

$$
\begin{aligned}
h_{11}= & \frac{\alpha G_{N} m_{2} m_{1}\left[(\varepsilon-1)(\varepsilon+3) \csc ^{2}\left(\frac{E}{2}\right)+2\left(\varepsilon^{2}-3\right) \cos E\right]}{\cos E-1}, \\
h_{22}= & \frac{\alpha G_{N} m_{2} m_{1}\left[\left(2 \varepsilon^{2}+\varepsilon-3\right) \csc ^{2}\left(\frac{E}{2}\right)+\left(4 \varepsilon^{2}-6\right) \cos E\right]}{\cos E-1}, \\
h_{12}= & h_{21}=3 G_{N} m_{2} m_{1} \alpha \sqrt{-\frac{\left(\epsilon^{2}-1\right) \sin ^{2} E}{(\epsilon \cos E-1)^{2}}} . \\
& \frac{(\epsilon \cos E-1)(\epsilon-4 \cos E+\cos 2 E+2) \cot ^{2}\left(\frac{E}{2}\right) \csc ^{2} E}{\cos E-1}
\end{aligned}
$$

and then the expected strain amplitude $h$, which strictly depends on the initial conditions of the stellar encounter, can be evaluated in accordance to (11).

\section{B. Quadrupole and GW Amplitude from Hyperbolic Orbits}

When hyperbolic orbits are taken into account, Eq.s (5), (6) and (9) become:

$$
\begin{aligned}
Q_{x x} & =\alpha^{2} m_{1}(\epsilon \cosh F-1)^{2}\left[\frac{3(l+\alpha-\alpha \epsilon \cosh F)^{2}}{\left(\alpha \epsilon^{2} \cosh F-\alpha \epsilon\right)^{2}}-1\right] \\
Q_{y y} & =\alpha^{2} m_{1}(\epsilon \cosh F-1)^{2}\left[3\left(1-\frac{(l+\alpha-\alpha \epsilon \cosh F)^{2}}{\left(\alpha \epsilon^{2} \cosh F-\alpha \epsilon\right)^{2}}\right)-1\right] \\
Q_{z z} & =-\alpha^{2} \mu(\epsilon \cosh F-1)^{2} \\
Q_{x y} & =Q_{y x}= \\
& =\frac{3 \alpha \mu(\epsilon \cosh F-1)(l+\alpha-\alpha \epsilon \cosh F) \sqrt{1-\frac{(l+\alpha-\alpha \epsilon \cosh F)^{2}}{\left(\alpha \epsilon^{2} \cosh F-\alpha \epsilon\right)^{2}}}}{\epsilon}
\end{aligned}
$$

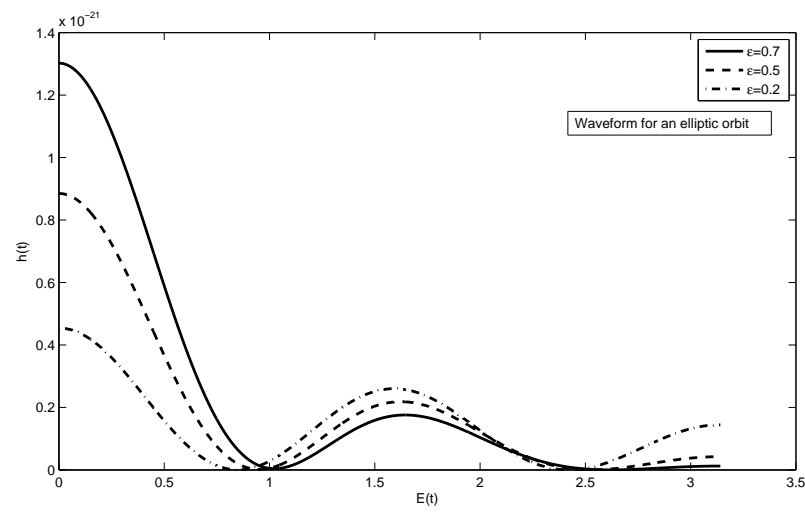

Fig. 1. The gravitational wave-forms from elliptical orbits shown as function of $E(t)$. We have fixed the masses of the order $1.4 M_{\odot} . m_{2}$ is considered at rest while $m_{1}$ is moving. The distance of the GW source is assumed to be $R=8 \mathrm{kpc}$ and the eccentricity is $\varepsilon=0.2,0.5,0.7$.

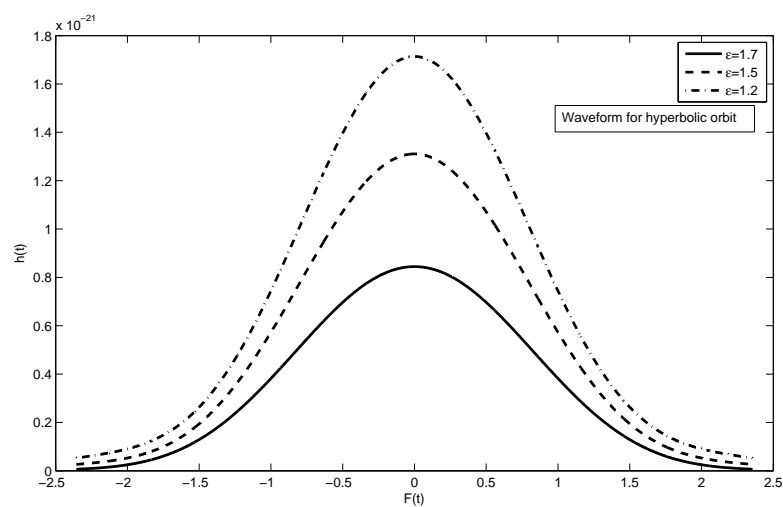

Fig. 2. The gravitational wave-forms for hyperbolic encounters as function of the $F(t)$. As above, we have fixed the masses of the order $1.4 M_{\odot} . m_{2}$ is considered at rest while $m_{1}$ is moving. The distance of the source is assumed at $R=8 \mathrm{kpc}$. The eccentricity is assumed with the values $\varepsilon=1.2,1.5,1.7$

In this case the single components of Eq. (10) for a hyperbolic orbit, are:

$$
\begin{aligned}
h_{11}= & \frac{2 G_{N} m_{2} m_{1}}{\varepsilon^{2}}\left[\frac{3 l^{3}}{\alpha^{2}(\varepsilon \cosh F-1)^{3}}-\frac{9 l^{2}}{\alpha(\varepsilon \cosh (F(t))-1)^{2}}+\right. \\
& \left.+\frac{\left(9-2 \varepsilon^{2}\right) l}{\varepsilon \cosh F-1}+\alpha\left(-2 \varepsilon^{4}+8 \varepsilon^{2}-3\right)\right], \\
h_{22}= & \frac{2 G_{N} m_{2} m_{1}}{\varepsilon^{2}}\left[-\frac{3 l^{3}}{\alpha^{2}(\varepsilon \cosh F-1)^{3}}+\frac{9 l^{2}}{\alpha(\varepsilon \cosh F-1)^{2}}\right. \\
& \left.+\frac{\left(4 \varepsilon^{2}-9\right) l}{\varepsilon \cosh F-1}+\alpha\left(4 \varepsilon^{4}-10 \varepsilon^{2}+3\right)\right], \\
h_{12}= & h_{21}=-\frac{2 G_{N} m_{2} m_{1}\left(\frac{(l+\alpha-\alpha \varepsilon \cosh F)^{2}}{\alpha^{2} \varepsilon^{2}(\varepsilon \cosh F-1)^{2}}+1\right)^{3 / 2}}{\alpha \varepsilon(\varepsilon \cosh F-1)^{2}} . \\
l^{2}+ & \alpha^{2}\left(\varepsilon^{2}-1\right)+\alpha^{2} \varepsilon\left(\varepsilon^{2}-1\right) \cosh F(\varepsilon \cosh (F-2)),
\end{aligned}
$$

which, as before, strictly depends on the initial conditions of the stellar encounter. 


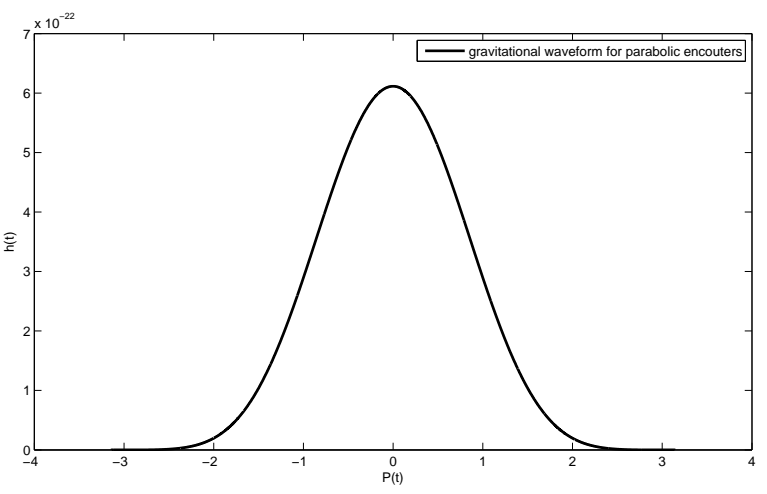

Fig. 3. The gravitational wave-forms for a parabolic encounter as a function of $P(t)$. As above, we have fixed the masses of the order $1.4 M_{\odot}$ and $m_{2}$ is considered at rest, while $m_{1}$ is moving. The distance of the GW source is assumed at $R=8 \mathrm{kpc}$. The eccentricity is $\varepsilon=1$.

\section{Quadrupole and GW Amplitude from Parabolic Orbits}

Finally, considering parabolic orbits, the explicit expression of the components of the quadrupole mass tensor in the equatorial plane through the parameter $P$ are deduced by (7), (8) and (9):

$$
\begin{aligned}
& Q_{x x}=\frac{1}{4}\left[\frac{3(2 l-1)^{2}}{P^{4}}-1\right] P^{4} m_{1}, \\
& Q_{y y}=\frac{1}{4} P^{4}\left\{\frac{3\left[P^{4}-(2 l-1)^{2}\right]}{P(t)^{4}}-1\right\} m_{1}, \\
& Q_{z z}=-\frac{P^{4} m_{1}}{4} \\
& Q_{x z}=Q_{z x}=0 \\
& Q_{y z}=Q_{z y}=0, \\
& Q_{x y}=Q_{y x}=\frac{3}{4}(2 l-1) P^{2} \sqrt{\frac{P^{4}-(2 l-1)^{2}}{P^{4}}} m_{1},
\end{aligned}
$$

Moreover, the single components of Eq. (10) for a parabolic orbit, are:

$$
\begin{aligned}
& h_{11}=-\frac{4 G_{N} m_{2} m_{1}}{P^{2}}, \\
& h_{22}=\frac{8 G_{N} m_{2} m_{1}}{P^{2}}, \\
& h_{12}=h_{21}=\frac{6 G m_{2} m_{1}\left[(1-2 l) P^{4}-(2 l-1)^{3}\right]}{\left[1-\frac{(1-2 l)^{2}}{P^{4}}\right]^{3 / 2} P^{8}},
\end{aligned}
$$

and then the expected strain amplitude $h$, which strictly depends on the initial conditions of the stellar encounter, can be evaluated in accordance to (11).

\section{CONCLUSION}

We have analyzed the gravitational wave emission coming from stellar encounters in Newtonian regime and in quadrupole approximation. In particular, we have taken into account the expected strain amplitude of gravitational radiation produced in tight impacts where two compact objects with masses comparable to the Chandrasekhar limit $\left(\sim 1.4 M_{\odot}\right)$. This choice is motivated by the fact that ground-based experiments like VIRGO or LIGO expect to detect typical GW emissions from the dynamics of these objects or from binary systems composed by them (see e.g. [12]). We would like to underline that the presented approach provides the necessary support for the application of computational schemes to obtain qualitative description of the Gravitational Waves.

\section{ACKNOWLEDGMENT}

The authors would like to thank Prof. Salvatore Capozziello for his very useful discussions and suggestions which allowed us to improve the paper.

\section{REFERENCES}

[1] A.M. Ghez, B.L. Klein, M. Morris, and E.E. Becklin Ap. J., Vol 509, 678 (1998).

[2] P.C. Peters and J. Mathews Phys. Rev. Vol 131, 435 (1963).

[3] P.C. Peters Phys. Rev. 136, 1224 (1964).

[4] S. Capozziello S. and M De Laurentis, Gravitational waves from stellar encounters Astropart.Phys., Vol 30, 105-112 (2008).

[5] B.F. Schutz Class. Quantum Grav. Vol. 16, 131 (1999).

[6] I. Bochicchio and E. Laserra, Evolution of dust shells in Tolman-Bondi space-time according to the Weierstrass approach in print on Journal of General Relativity and Gravitation.

[7] J. Binney and S. Tremaine, Galactic Dynamics Princeton University Press, Princeton, New Jersey, 1987.

[8] L. Landau and E.M. Lifsits, Mechanics Pergamon Press, New York, 1973.

[9] A.E. Roy, Orbital Motion, 3rd ed. Adam Hilger, 1988.

[10] C.W. Misner, K.S. Thorne and J.A. Wheeler, Gravitation Freeman, New. York, 1973.

[11] S.L. Shapiro and S.A. Teukolsy, Black Holes, White dwarfs and Neutron Stars Chicago Univ. Press, Chicago, 1983.

[12] M. Maggiore, Gravitational Waves, Volume 1: Theory and Experiments Oxford Univ. Press, Oxford, 2007.

[13] K.S. Thorne Rev. Mod. Phys., Vol. 52, 285 (1980).

[14] S. Capozziello, M. De Laurentis and M. Francaviglia Astrop. Phys., Vol. 29, 125 (2008). 